\title{
De la Perfection à la Cruauté (Analyse de Caligula de Camus)
}

\section{Şengül KOCAMAN ${ }^{\mathrm{a}^{*}}$}

\author{
${ }^{a}$ Dicle University, Ziya Gökalp Education Faculty, Diyarbakır/Turkey
}

\begin{tabular}{l}
\hline Article Info \\
\hline DOI: 10.14812/cufej.2014.008 \\
\hline Article history: \\
Received $\quad$ 14 January 2012 \\
Revised $\quad 23$ December 2013 \\
Accepted $\quad$ 05 February 2014 \\
\hline Keywords: \\
Camus, \\
Caligula, \\
Absurd, \\
Emperor. \\
\hline
\end{tabular}

\section{Abstract}

Camus' play Caligula written under the influence of the Roman historian Suetone's the Lives of Twelve Caesars deals with the rebellion of an absolute emperor of the whole Roman Empire against the fact that life ends with death. Undertaking the task of showing and teaching people the absurdness of such an unfair destiny, Caligula tells the adventure of a "fair", "honest", "helpful" and "artistic" emperor's transformation into a monster. Therefore, it is among Camus' one of the plays that reminds people of their temporary existence.

\section{Introduction}

C'est de La Vie des douze Césars de Suétone que Camus s'inspire pour écrire Caligula. La première version, sur laquelle Camus revient plusieurs fois, remonte à 1937, et la dernière, à 1958.

Caligula a été pensé comme une des parties de la trilogie que l'écrivain appelle lui-même « le cycle de l'absurde ", s'insérant entre L'Étranger et Le Mythe de Sisyphe. Grâce à des genres différents, Camus a approfondi la notion qui était, avant la guerre, au cœur de sa réflexion philosophique. Homme de théâtre par vocation, il indique ainsi que la scène lui offrait un moyen d'illustrer ses idées.

Dans cette pièce, l'auteur met en scène la tragédie d'un homme: un empereur assoiffé de pouvoir sans limites, un Romain tyrannique qui impose la logique d'un empereur fou. En somme, nous voilà confrontés à un roi qui parle follement d'un besoin d'impossible. Pourquoi et comment un roi arrive-t-il à un tel désir et une telle pulsion? Comment un tel empereur " parfait » et " aimable » en vient-il à devenir " cruel ", " meurtrier », " atroce " et même « fou » ?

Albert Camus et son théâtre ont déjà fait l'objet d'un flot de critiques. Mais malgré de multiples études, le théâtre de Camus mérite encore que l'on s'y intéresse, surtout que Caligula semble être la production dramaturgique la plus riche de Camus. Au-delà de la pièce, analysons plutôt ce curieux protagoniste si ambigu de plus près, ainsi que les relations qu'il entretient avec les autres personnages.

\section{Caligula Avant La Mort De Drusilla}

\section{(Un empereur « aimable », " un empereur parfait »)}

Le Premier Patricien: Pourquoi s'inquiéter à l'avance? Attendons; il reviendra peut-être comme il est parti.

*Corresponding author: senkocaman@hotmail.com 
Le Vieux Patricien: Je l'ai vu sortir du palais. Il avait un regard étrange (Camus, 1958, p. 16).

La pièce débute par l'attente des patriciens qui dure depuis trois jours, et l'inquiétude est au rendezvous. En effet, voici le contexte dans lequel le dramaturge nous plonge: l'empereur est parti sans laisser d'explications. Les dialogues échangés entre les patriciens amènent le jugement du spectateur à se focaliser sur le passé du roi; les deux premières scènes de l'acte premier font appel à ce qui se passait avant le début de la pièce. Quelles qualités peuvent susciter un tel intérêt pour un empereur?

Caligula est, comme le présente Camus pour l'édition américaine de sa pièce, " un prince relativement aimable jusque-là [...] et Son règne commença d'ailleurs, dans la douceur et la sagesse " (Camus, 1962, p. 1727). En fait, avant la mort de Drusilla, son règne était bien accueilli; on disait même qu'il entretenait de bonnes relations avec son peuple. C'est donc avec grand regret que Cherea annonce : «Mais tout allait trop bien. Cet empereur était parfait » (Camus, 1958, p. 18) ; "Ce garçon aimait trop la littérature. [...] Un empereur artiste » (Camus, 1958, p. 21).

Homme de lettres et intellectuel, Cherea se distingue non seulement par ses qualités d'érudit, mais aussi par sa proximité avec l'empereur; Caligula le prend en effet comme confident et récompense son savoir en le qualifiant même de "littérateur ».

La distance entre Caligula et Cherea jalonne deux âges de la vie, deux aspects opposés de Camus, deux époques dans sa réflexion. Lorsque la pièce fut créée en 1945, il se sentait en harmonie avec I'humanisme de Cherea et dans ses commentaires il fera le procès de l'empereur fou. [...] Mais quand Camus méditait sur sa pièce en 1938, c'est évidemment la frénésie de Caligula qui l'intéressait (Onimus, 1965, p. 79).

Le discours de Cherea brosse le portrait d'un empereur doux et réfléchi. Notamment grâce à ce personnage, la poésie tient une place importante dans la pièce. Sa réplique « j'aimerais mieux qu'on me laisse à mes livres "(Camus, 1958, p. 22) laisse deviner sa passion pour la culture et son niveau d'intellect. Un autre personnage important, Scipion, est caractérisé par son statut de " poète ". La scène du concours de poésie organisé par Caligula met en évidence la valeur de la poésie dans la pièce et laisse deviner que Camus voulait mettre en avant l'érudition littéraire dans son œuvre.

Plusieurs voix rappellent au cours de la pièce que Caligula est délicat, généreux, juste, comme le dit le jeune poète Scipion:

Je l'aime. Il était bon pour moi. II m'encourageait et je sais par cœur certaines de ses paroles. Caligula disait que la vie n'est pas facile, mais qu'il y avait la religion, l'art, l'amour qu'on nous porte. II répétait souvent que faire souffrir était la seule façon de se tromper. Il voulait être un homme juste (Camus, 1958, p. 30).

Ces propos donnent le ton de la pièce: Caligula a donc jadis vu l'art, la religion ou l'amour comme un refuge contre la réalité. II trouvait que l'art, la religion et l'amour étaient des remèdes suffisants contre la douleur de la condition humaine. Plus loin, dans la scène 6 , on est encore mis au courant d'autres qualités de Caligula; Scipion fait apparaître les diverses facettes du personnage: un roi qui réussissait à attirer la confiance et l'attention du palais, il était tendre et même altruiste: " II répétait souvent que faire souffrir était la seule façon de se tromper ". Quant à Hélicon, un autre patricien, il voit en lui un idéaliste:

Mais si Caius se met à comprendre, ajoute-t-il, il est capable au contraire, avec son bon petit cour, de s'occuper de tout (Camus, 1958, p. 29). 
L'ensemble des propos dont le lecteur est témoin dans les premières scènes de l'acte I révèle que c'est la disparition de l'empereur, à la suite de la mort de Drusilla, qui bouleverse l'équilibre parfait qui régnait jusqu'alors. Comment un roi si sympathique et recueillant autant de qualités peut-il devenir " cruel » voire « criminel » ? A ce propos, Chapoutot (2006, p.4) explique que:

Caligula n'est pas d'essence criminelle, même s'il en a adopté l'existence. Pour que l'homme qu'il était ait fait un tel choix et soit devenu l'empereur insensible et cruel que nous connaissons et voyons, il faut qu'il y ait eu rupture, une catastrophe fondatrice, inaugurale, que le théâtre met en scène.

La catastrophe en question est bien sûr la mort de Drusilla, et c'est sur cette catastrophe que s'ouvre la pièce débutant par ces deux mots : "Toujours rien » (Camus, 1958, p.20). Une telle ouverture laisse deviner l'atmosphère d'inquiétude et de désespoir qu'a laissé Caligula derrière lui. La quête pour le retrouver reste vaine. L'État a perdu son chef. Scipion témoigne qu'avant sa fuite, il était comme tétanisé:

Il s'est avancé vers le corps de Drusilla. II l'a touché avec deux doigts. Puis il a semblé réfléchir, tournant sur lui-même, et il est sorti d'un pas égal. Depuis, on court après lui (Camus, 1958, p. 20).

Caligula a touché le cadavre de sa bien-aimée Drusilla, puis il se retire en chancelant et disparaît.

Quand il reviendra au palais impérial ce ne sera plus le même homme: il a fait la rencontre du Mal. Les mots qu'il prononce alors sont très simples mais lourds de signification (Onimus, 1965, p. 76).

La mort, fait universel, provoque un choc existentiel chez l'empereur. Devant le cadavre de sa dulcinée, il découvre brusquement que « ce monde tel qu'il est fait n'est pas supportable » (Camus, 1958, p. 26). Le monde n'offre aucun espoir. La mort de Drusilla l'amène à la conscience de cette vérité. Le palais estime que cette mort l'a choqué et égaré. Mais en fait, ce n'est pas la mort de Drusilla qui le fait agir et qui le pousse vers cette fuite. Il a plutôt compris que tout " autour de lui est mensonge ", il souffre de l'évidence d'une vérité de la vie : "Les hommes meurent et ils ne sont pas heureux ", (Camus, 1958, p. 27) dit-il, ajoutant que la mort de Drusilla " n'est rien, elle est seulement le signe d'une vérité " (Camus, 1958, p. 26). Cet événement incontournable de la vie lui fait conclure que le monde est sans importance et que le bonheur demeure inaccessible à l'Homme.

Le souvenir de Drusilla occupera une place toute secondaire et Caligula sera rongé intérieurement non plus par sa perte personnelle mais par la mort universelle devenue pour lui un scandale métaphysique (Clayton, 2009, p. 166 cité par Guerry, 2009, p.8).

La vérité de la mort universelle est une fatalité que les gens essayent de contourner, mais finalement cela ne les rend pas plus heureux. Ils meurent en ignorant qu'ils n'ont jamais été heureux. La mort de Drusilla met en marche l'action de la pièce, et la transformation de Caligula.

\section{Caligula Après La Mort De Drusilla}

\section{(Le déclin et la colère d'un empereur frustré)}

Caligula s'enfuit et erre, désespéré, pendant trois jours. Lorsqu'il revient au palais, il est complètement métamorphosé. On remarque déjà des changements physiques et d'allure; il est hagard, incohérent, presque halluciné. II reparaît souillé et égaré après trois jours d'errance. Ce changement est bien précis dans les didascalies:

Il a l'air égaré, il est sale, il a les cheveux pleins d'eau et les jambes souillées. II porte plusieurs fois la main à sa bouche. Il avance vers le miroir et s'arrête dès qu'il aperçoit sa propre image. Il grommelle des 
paroles indistinctes, puis va s'asseoir, à droite, les bras pendants entre les genoux écartés (Camus, 1958, p. 23).

Caligula lui-même constate son état physique en regardant sa propre image dans le miroir : «je suis fatigué... » (Camus, 1958, p.23-25). Ces changements ne sont pas visibles uniquement au niveau de son apparence, mais également au niveau psychique et corporel. La métamorphose de Caligula est complète:

Caligula: C'est le corps qui souffre. Ma peau me fait mal, ma poitrine, mes membres. J'ai la tête creuse et le cœur soulevé. Et le plus affreux, c'est ce goût dans la bouche. Ni sang, ni mort, ni fièvre, mais tout cela à la fois. II suffit que je remue la langue pour que tout redevienne noir et que les êtres me répugnent. Qu'il est dur, qu'il est amer de devenir un homme! (Camus, 1958, p. 39-40).

A 24 ans, c'est un roi bien jeune qui se trouve « désespéré » et qui doit faire face au lourd fardeau de " devenir un homme ». II mentionne d'ailleurs à plusieurs reprises la dure réalité à laquelle il fait face. II confie notamment à Hélicon : "Alors c'est que tout, autour de moi, est mensonge " (Camus, 1958, p. 27). Par la suite, Caesonia l'entend même dire : « Les hommes pleurent parce que les choses ne sont pas ce qu'elles devraient être " (Camus, 1958, p. 39).

La réplique de Caligula est le signe de la découverte de la relation ridicule qu'entretient l'Homme avec le monde. La prise de conscience de l'empereur est son point de départ. Sa révolte fait suite à cette révélation, naissance en lui du " sentiment de l'absurde » que Camus définit dans le Mythe de Sisyphe : "L'absurde naît de cette confrontation entre l'appel humain et le silence déraisonnable du monde " (Camus, 1985, p. 46). La vie vaut-elle la peine d'être vécue? Telle est la question existentielle que fait valoir Caligula et qui amorce le scandale qui s'ensuit. Est-il possible de vivre après avoir pris conscience de l'absurde? La réponse s'avère positive. Le personnage camusien répond non par le suicide, mais oui par la révolte. Sa vie se construit désormais dans une direction bien particulière: en se révoltant contre ce qu'il a jugé d'absurde. Caligula postule alors que la vérité est de se révolter contre le destin, et ce désir de révolte le conduit à un désir d'impossible et d'absolu: dans la scène 3 de l'acte I, au moment de son retour, il confie à Hélicon qu'il a erré car il voulait la lune, métaphore emblématique de l'impossible.

Ce monde, tel qu'il est fait n'est pas supportable. J'ai donc besoin de la lune, ou du bonheur, ou de l'immortalité, de quelque chose qui soit dément peut-être, mais qui ne soit pas de ce monde (Camus, 1958, p. 26).

Nous assistons à une démonstration de l'esprit contradictoire de Caligula: il se déclare raisonnable au point de répéter: " je ne suis pas fou » (Camus, 1958, p. 25); il se rend compte que le monde est déraisonnable : " les hommes meurent et ne sont pas heureux "; il décide d'affronter ce problème, situation contradictoire, par l'acquisition de l'impossible. Pour lui, la vie est absurde, alors il sera aussi absurde que la vie. Ainsi, il demande la lune, veut requérir l'impossible. La vie lance un défi à chaque homme, et Caligula décide de regagner de sa causalité sur la vie en la défiant à son tour.

Caligula veut la lune pour assouvir un besoin de "bonheur " et " d'immortalité ", pour surmonter l'absurdité de l'existence et du monde; il témoigne de sa révolte en exprimant le besoin de quelque chose qui soit dément peut-être, mais qui ne soit pas de ce monde (Hobby, 1998, p. 158).

S'amorce alors une lutte contre l'incompréhensibilité du monde d'une manière déraisonnable et irrationnelle. Une quête extraordinaire et fantasque est menée: dès lors, Caligula refuse de se soumettre au destin en choisissant de se faire cause du destin. II détruit et tue tout autour de lui. " II décide de tirer la logique extrême de sa révolte en la transformant en tyrannie, de vivre sa liberté aux dépens de celle des autres, la pièce devient un drame sanguinaire " (Durand, www.comptoirlitteraire.com, p.5). Ainsi les hommes prendront-ils conscience de l'horreur de leur 
condition et se révolteront en se soulevant contre lui. II s'institue pédagogue du genre humain en avançant qu' " ils sont privés de la connaissance et il leur manque un professeur qui sache ce dont il parle "(Camus, 1958, p. 27). Son peuple doit être amené à se rebeller contre l'absurde. II va agir de manière à montrer la vanité de ce monde, la vérité de sa condition insupportable et inacceptable. Comme le dit Cherea : "il force tout le monde à penser "(Camus, 1958, p. 123). Mais comment qualifierait-on raisonnablement un empereur qui se lance dans le massacre de son peuple pour lui faire ouvrir les yeux? Serait-il au fond un fin et dévoué pédagogue ou plutôt un tyran meurtrier? En découvrant que " les hommes meurent et [qu'] ils ne sont pas heureux » (Camus, 1958, p. 27), il choisit de faire vivre l'absurde et d'être maître du destin. Mais de quel droit un roi peut-il décider du destin de son peuple? Est-ce là sa fonction première? Est-ce la meilleure façon d'œuvrer à la protection de celuicí? À ce sujet, Raymond Gay-Crosier affirme que "Caligula est un Sisyphe parvenu dont le problème n'est pas de s'accommoder d'un destin mais de l'infliger à autrui » (1992). Pour sa révolte contre la mort, sa passion pour la vie et son mépris des dieux, Caligula est bien l'homme absurde et le frère du Sisyphe de la légende:

Caligula ajoute au problème du comportement de l'homme absurde une autre dimension, celle de sa responsabilité envers les êtres. Par ailleurs, la légende de Sisyphe illustre un style de vie absurde, mais non point la puissance destructrice de la pensée absurde poussée jusqu'à ses ultimes conséquences. Caligula est la tragédie d'une révolte généreuse et d'un désir de bonheur justes, au départ, qui s'achèvent dans la destruction, ajoutant ainsi au mal qu'il voulait combattre (Crochet, 1973, p. 148149).

Une logique imperturbable apparaît alors. D’abord, il s'agit de détruire certaines valeurs individuelles comme l'amour ou l'amitié et puis des valeurs sociales comme la religion et la littérature.

\section{Les Valeurs Détruites Par Caligula}

Caligula, " prince relativement aimable ", s'aperçoit que le monde n'est pas satisfaisant. II récuse alors l'amitié, l'amour, la simple solidarité humaine. Il laisse libre cours à la perversion de toutes les valeurs de sa société. Et ce ne sont pas les valeurs acceptées ou défendues par les patriciens qui l'entourent, mais des valeurs universelles. André Durand qualifie ainsi Caligula qui subit une mutation complète : " en tout cas, déçu, malheureux, éperdu, désespéré, cet ange désormais déchu devient délinquant, méchant, rageur, détestable, d'une versatilité et d'une imprévisibilité constantes...» (Durand, www.comptoirlitteraire.com, p. 13).

\section{-L’amour (Caligula et Caesonia)}

L'amour, valeur intemporelle et universelle, est dévalorisé par le roi. Caesonia, la maîtresse de Caligula, sa confidente qui lui avoue toujours son amour et qui montre toujours son côté protecteur et maternel est étranglée par son amant. Cette femme, définie comme " la vieille maîtresse " de l'empereur, essaye de ramener Caligula à l'amour, sentiment qu'elle évoque à plusieurs reprises : "Tu ne pourras nier l'amour " (Camus, 1958, p. 41) ; "Je n'ai pas besoin de jurer, puisque je t'aime "; " Fais alors servir ton pouvoir à mieux aimer ce qui peut l'être encore. Viens. Etends-toi près de moi. Mets ta tête sur mes genoux " (Camus, 1958, p.41-42). En réponse à une telle déclaration d'amour, Caligula parle de «tendresse honteuse » en dévalorisant totalement le concept de l'amour:

L'amour, Caesonia, j'ai appris que ce n'était rien. Vivre, Caesonia, vivre, c'est le contraire d'aimer. L'amour ne m'est pas suffisant (Camus, 1958, p. 146).

Caesonia ne cesse pour autant d'évoquer son amour, le supplie, mais Caligula propose d'autres choses, contraires. Il fait entendre sa tyrannie, son incontrôlable soif de pouvoir et vengeance: 
Caesonia: Mais arrête.

Caligula: Tu feras tout ce que je te dirai. [...] Tu seras cruelle, froide, et implacable (Camus, 1958, $p$. 42-43).

Epouvantée mais impuissante face à cet homme qu'elle aime, elle constate que son amant souffre et pense qu'elle pourra l'aider. La consolation est-elle possible? Elle essaye du moins, bien qu'il réfute sans cesse ses propos et s'oppose ainsi à elle. Caesonia n'a donc plus aucun pouvoir sur lui et assiste, désarmée, à sa métamorphose en roi fou et elle finira cruellement étranglée par l'empereur.

\section{L'amitié (Caligula et Scipion)}

Cette valeur aussi est tournée en dérision par le roi. Dans la pièce, l'amitié a été symbolisée par le lien qui l'unit à Scipion. Avant la mort de Drusilla, Caligula montrait de l'intérêt et de l'amour pour la poésie qui le faisait communier avec le jeune poète.

Ce personnage se caractérise par sa fidélité à Caligula. Trois ans auparavant, Caligula avait tué le père de Scipion, et malgré la haine que cela a pu susciter chez ce dernier, il refuse de se mêler au complot organisé par les patriciens pour le tuer. Parce qu'il le craint? Non, il comprend et adopte vraiment la logique désespérée de son empereur, lui-même victime de la même idée. Lui aussi a découvert le caractère absurde de la vie au moment où il a perdu son père. Caligula dit lui-même : " Quelque chose en moi lui ressemble pourtant. La même flamme nous brûle le cœur » (Camus, 1958, p. 118). De plus, Scipion exprime son respect pour les croyances des autres, il défend la liberté individuelle : "Je puis nier une chose sans me croire obligé de la salir ou de retirer aux autres le droit d'y croire " (Camus, 1958, p. 93). Il préfère toujours être honnête et parle franchement avec l'empereur, et surtout n'hésite pas s'opposer à sa démesure et l'accuse même d'être un tyran : "Quel cœur ignoble et ensanglanté tu dois avoir. [...] Comme je te plains et comme je te hais. [...] Et quelle immonde solitude doit être la tienne! » (Camus 1958, p. 81-82).

L'entourage de Caligula est plein de patriciens flatteurs, mais il s'agit là d'une flatterie cultivée dans la peur. Scipion, lui, échappe à ces caractéristiques. Il ne ment pas et se distingue des autres par cette particularité. Caligula le remarque : "Tu es pur dans le bien comme je suis pur dans le mal » (Camus, 1958, p. 81). L'empereur résume bien leurs sentiments par cette belle formule; ces deux êtres sont effectivement les deux faces d'une même médaille, ils sont frères dans le lyrisme.

Ainsi, Scipion est l'unique personnage à voir Caligula au naturel, à lui faire renoncer à son masque, à le sortir de son jeu, à tel point qu'il faut même que Caesonia le rappelle à l'ordre : "Comme tu y vas mon garçon; il y a en ce moment, dans Rome, des gens qui meurent pour des discours beaucoup moins éloquents " (Camus, 1958, p. 93). Scipion est donc un personnage fort ; il comprend l'empereur et se met à ses côtés pour se lancer dans le même combat. Mais la haine qu'il entretient en contrepartie pour son roi fait de lui un personnage qui tient tête à l'autorité, et Caligula, tenant parfaitement son rôle despotique, ne peut tolérer tant de résistance. II met fin à cela, et au passage à l'amitié qui le liait au poète. C'était d'ailleurs son dernier ami.

Caligula: Scipion est parti. J'en ai fini avec l'amitié (Camus, 1958, p. 141).

\section{Rejet de l'art (Caligula et Cherea)}

Dès son retour à Rome au premier acte, Caligula qui aimait trop la littérature ne voit plus la création littéraire de la même manière. II se montre brusque et précise qu'il considère désormais la littérature comme un tissu de mensonges. Voici un dialogue entre Cherea et Caligula qui met en évidence le rejet de la littérature par l'empereur: 
Cherea: Si nous mentons, c'est souvent sans le savoir. Je plaide non coupable. [...] Et pourtant, il faut bien plaider pour ce monde, si nous voulons y vivre (Camus, 1958, p. 37).

J'ai envie de vivre (Camus, 1958, p. 109).

Pour Cherea, la littérature témoigne du monde dans lequel vit l'artiste et lui donne de la valeur. Mais pour Caligula, rien n'a de valeur, l'unique évidence est que ce monde est sans importance.

Caligula: Ne plaide pas, la cause est entendue. Ce monde est sans importance et qui le reconnaît conquiert sa liberté. [...] Et justement, je vous hais parce que vous n'êtes pas libres. [...] Réjouissez-vous, il vous est enfin venu un empereur pour vous enseigner la liberté (Camus, 1958, p. 38).

Maintenant, Caligula hait les littérateurs qui ne sont pour lui que de " faux témoins », il les traite comme des menteurs, et l'art comme impuissant. II prend conscience qu'il n'existe aucun refuge alors qu'auparavant, il voyait l'art comme un refuge contre la réalité. À son retour, s'il détruit tout ce qu'il a autrefois considéré comme un refuge, même la poésie, c'est parce qu'il a pris conscience du fait que ni la littérature, ni l'amour, ni aucune autre valeur dans la vie n'étaient capables de trouver une solution pour que les gens soient heureux. II nie toutes les valeurs que nous venons d'énumérer. II refuse logiquement d'accorder de la valeur à quoi que ce soit. En s'adressant à Cherea, l'empereur souligne et répète à plusieurs reprises que la littérature n'est qu'un mensonge.

Heureusement face à Caligula il y a Cherea, l'homme mûr qui, non moins lucide que l'empereur adolescent (et donc non moins désespéré) a appris d'expérience ce qu'est la misère de l'homme et se refuse à y ajouter. Cherea sait qu'il y a des " limites » au-delà desquelles toute dignité disparaît. Caligula a le radicalisme de la jeunesse; pour lui, c'est tout ou rien... (Onimus, 1965, p. 78).

Face à Caligula qui choisit les valeurs de la mort, Cherea affirme les valeurs de la vie. La formulation de Cherea est exactement antithétique à celle de son roi. A l'axiome de celui-ci « ce monde est sans importance ", Cherea oppose son désir " j'ai envie de vivre ", ce qui prouve qu'il reconnaît l'absolu de la mort mais tente de le dépasser, et l'art est l'un des moyens d'y parvenir. Cela ne veut pas dire que Cherea élude complètement l'idée de la mort mais il l'affronte avec sang-froid et refuse la liberté totale que Caligula se permet, parce que cette pseudo-liberté nie le monde et l'Homme. Pour Cherea, la liberté totale est une folie qui aboutit à la mort. En bref, Cherea voit en son empereur l'élément qui trouble l'ordre de la société, et il refuse de le comprendre : « Je te juge nuisible » (Camus, 1958, p. 108).

\section{La religion}

"Caligula est I'histoire d'un homme qui a poussé jusqu'au bout sa contestation de la création divine» (Toura, 1992, p. 29). Est-il athée comme il le dit souvent? Caligula est bien loin de l'athéisme nietzschéen. II n'a pas l'intention de détruire les dieux, ou de les chasser de ce monde comme dans Le Mythe de Sisyphe, il les accuse d'avoir créé l'Homme avec comme condition irrévocable l'immortalité. " Il est peu probable que l'empereur romain croie en ces dieux, mais il agit comme s'ils existaient. Tout en les qualifiant d'illusoires, il les tient pour responsables de ce monde » (Toura, 1992).

La représentation classique du Dieu de l'Ancien Testament est Dieu le père, il incarne donc un rôle paternel, mais c'est aussi un dieu qui use de sa colère et de son pouvoir de justice, soumettant les hommes aux lois, devoirs et même punition; ils lui doivent ainsi naturellement respect et obéissance. Ici en revanche, Caligula nous fait part d'un point de vue quelque peu différent: les dieux agiraient et interviendraient dans le destin des individus. II accuse par ailleurs les dieux pour leur création principale: les hommes sont mortels, une loi inacceptable et insupportable pour l'empereur. Comme l'affirme Pierre Nguyen-van-Huy (1962, p.61): "Caligula n'est pas à proprement parler une application des canons nietzschéens, mais une réaction contre leurs formules extrêmes et leurs conséquences absurdes». 
Torturé par la douleur de l'absurdité de la vie, le roi se jette dans la quête de l'impossible qu'il mène jusqu'au point de remplacer les dieux. A l'acte III, une bouffonnerie grotesque commence. Les patriciens sont forcés de répéter une prière d'adoration à Vénus qui n'est autre que Caligula travesti.

Caligula en robe courte de danseuse, des fleurs sur la tête, paraît en ombre chinoise, derrière le rideau du fond, mime quelques gestes ridicules de danse et s'éclipse (Camus, 1958, p. 124).

« La religion est elle aussi discréditée, par la parodie de prière de l'acte III, scène 1, le registre burlesque est utilisé pour évoquer l'Olympe, et par le déguisement de Caligula en Vénus grotesque " (Houel, 2006, p. 64). Le roi vise plutôt par son déguisement à railler les dieux, alimentant le ridicule par le caractère contradictoire de la scène: il est habillé en Vénus dans le morne contexte que représente la pièce peuplée de morts. Son déguisement comique et moqueur démontre le rejet du pouvoir divin, mais également la folie dont il est atteint. II ne relâche aucun effort pour tenter de dépasser les dieux. Même s'il n'accorde plus de valeur à la création artistique, il utilise ici l'art dramatique pour enseigner sa logique aux autres, pour démontrer les conséquences de l'absurde. Mais ce dont Caligula a envie, ce n'est même pas leur puissance, c'est plutôt leur nature incompréhensible.

On ne comprend pas le destin et c'est pourquoi je me suis fait destin. J'ai pris le visage bête et incompréhensible des dieux (Camus, 1958, p. 96).

"Le jeune homme montre en quelle pieuse estime il tient des dieux incapables de prévenir le scandale de la mort, qu'il ridiculise dans une mascarade carnavalesque " (Chapoutot, 2006, p. 8). Le spectacle est annoncé comme " un miracle sacré » mais il s'agit plutôt d'une satire du pouvoir des dieux. Caligula accuse les dieux; ils sont responsables d'avoir créé les hommes comme étant mortels et de les avoir mis en ce bas monde, et cela est insupportable.

Pourquoi Vénus? C'est un choix important. Vénus est une déesse et demeure la symbolique par excellence de la féminité; elle règne sur la fécondité et la procréation, c'est donc elle qui régit la naissance des hommes. De ce point de vue, ce sujet se prête à une telle interprétation de Toura (1992, p.32) " il s'agit, premièrement, de s'enfuir dans le fantasme du retour au paradis prénatal, et deuxièmement, de détruire la menace de l'hostilité maternelle ". Le retour au paradis prénatal est un refuge, un moyen de s'éloigner des réalités insupportables de la vie. Mais surtout, Caligula accuse Vénus, et pas seulement les dieux masculins qui, comme nous l'avons vu plus haut exigent obéissance et respect. Bien qu'elle soit une femme, et représente même la maternité, Vénus n'en est pas moins hostile aux yeux de l'empereur: elle a particularité de faire don de fécondité, elle permet donc de mettre des êtres humains au monde, ce qui est une bien grande supercherie étant donné la condamnation inévitable qui attend chaque être en fin de vie.

En fait, Caligula a découvert ce que tout un chacun savait déjà de façon passive quant à la mort et la condition mortelle de l'Homme. "Tout le projet prêté à Caligula est donc de passer - et de faire passer son entourage - de ce savoir passif (ce que chacun sait) à un savoir actif. Pour y parvenir, Caligula s'est « fait destin " en s'efforçant d'offrir "le visage bête et incompréhensible des dieux en mimant leur comportement arbitraire » (Bartfeld, 1988, p. 53).

\section{La vie humaine}

Caligula ne se contente pas seulement de détruire les valeurs acceptées, il devient de plus en plus un assassin qui exécute toutes sortes de tortures et d'exécutions. Il dévore et détruit tout ce qui l'entoure. II se renferme dans un monde où l'amitié, l'amour et même la vie humaine inversent leurs polarités et ne provoquent que la révolte. 
L'empereur fou ridiculise ses patriciens, en les « faisant courir tous les soirs autour de sa litière "; il les insulte, leur confisque leurs biens, prend leurs femmes, comme celle de Murcius; et jusque sous leurs yeux, tue leurs proches, les force à rire de leurs souffrances, comme Lépidus dont il a tué le fils. En bref, la vie humaine est considérée par Caligula comme négligeable et réduite à du chaos. Il dit à l'intendant:

Tous les patriciens, toutes les personnes de l'Empire qui disposent de quelque fortune - petite ou grande, c'est exactement la même chose - doivent obligatoirement déshériter leurs enfants et tester sur I'heure en faveur de l'État. [...] À raison de nos besoins, nous ferons mourir ces personnages dans l'ordre d'une liste établie arbitrairement (Camus, 1958, p. 33).

Caligula se transforme en un César brutal, déroutant, imprévisible et cruel au point qu'il tue violemment Mereia. La scène 10 de l'acte II est assez importante, elle permet de considérer à quel point Caligula joue avec la vie humaine: l'empereur regarde le vieil asthmatique boire une gorgée de son remède en le soupçonnant d'avoir bu du contrepoison. Il lui reproche de s'être opposé à sa volonté et force Mereia à avaler du poison. Il finit évidemment par tuer le pauvre malade. La cruauté de Caligula se retrouve essentiellement dans ses propos:

Caligula: Tu crains que je ne t'empoisonne. Tu me soupçonnes. Tu m'épies (Camus, 1958, p. 70).

Tiens... Bois ce poison.

Mereia, secoué de sanglots, refuse de la tête (Camus, 1958, p. 72).

Pour l'empereur tout est équivalent, tout est positionné à une égalité absolue. "Tout est important [...], tout est capital [...], tout est sur le même pied: la grandeur de Rome et tes crises d'arthritisme " (Camus, 1958, p. 32), dit Caligula à Caesonia. Puisque tout est mortel, rien ne possède de valeur. Pas même la vie humaine. Caligula n'accorde plus d'importance à sa propre vie ni à celle d'autrui. « Un peu plus tôt ou un peu plus tard ", dit-il. Cette provocation pousse les patriciens à passer à l'action, il faut faire vite organiser réellement la conspiration contre ce roi qui a totalement perdu toute sa raison et tout son humanisme.

\section{La Mort De Caligula}

\section{(Le monologue final devant le miroir)}

Le miroir est un accessoire important dans la scène finale. Seul devant son miroir, le roi parle à luimême. Le monologue de Caligula s'adresse à son reflet dans le miroir, il s'adresse à son double auquel il pose aussi des questions. Ici, le miroir est chargé d'une signification symbolique.

Les critiques de Caligula sont analysées par la fonction du miroir en tant que le révélateur psychologique. L'accessoire est employé pour rendre mélancolique selon Albert Mingelgrün, le désespoir selon Janine Gillis, schizophrénique selon Georges Bauer et la paranoïa selon Léonard Henry Robbins. Un dédoublement de la personnalité s'effectue quand Caligula se regarde, commente James Arnold (Bastien, 2006).

Caligula pleure et s'agenouille devant son miroir, parce qu'il a échoué dans sa quête. Il reconnaît son erreur dans sa quête de l'impossible, dans son exigence d'absolu symbolisé par la lune. « Je n'ai pas pris la voie qu'il fallait, je n'aboutis à rien. Ma liberté n'est pas la bonne » (Camus, 1958, p. 149). Ces trois phrases négatives et l'adverbe " rien » soulignent bien son aveu d'échec total. Devant cet objet, il fait un bilan de sa vie et analyse les raisons de son échec. Il a réussi à détruire toutes les valeurs qui semblaient exister autour de lui, il a supprimé des êtres qui l'avaient aimé (Scipion: parti; Caesonia: étranglée). II constate enfin que tuer n'est pas la solution. 
Quant à son comportement suicidaire dans la scène 3 de l'acte III, Caligula refuse d'entendre raison lorsque Hélicon lui signale le complot qui se trame contre lui. Exaspérés par la folie de l'empereur, les patriciens préparent un coup d'État, mais il choisit librement de laisser les comploteurs l'assassiner. II sait bien que c'est à cause de ses actes que les patriciens organisent son assassinat, il en est bien conscient et les laisse faire. Il sait qu'en niant son peuple il s'est lui-même nié aussi. Le despote de Camus aurait-il retrouvé la raison en faisant face à la réalité grâce au miroir? Ainsi, déciderait-il donc de payer pour ce qu'il a infligé? Comme nous l'avons déjà précisé, le protagoniste ne répond pas par le suicide. L'homme absurde est celui qui demeure dans l'absurde, mais Caligula choisit d'ignorer le complot et consent à son propre assassinat. Pour apporter un peu d'analyse à sa propre œuvre, Camus avance que "Caligula est l'histoire d'un suicide supérieur " dans la préface de l'édition américaine. Qu'entend-il par « le suicide supérieur »?

C'est l'histoire d'un suicide supérieur. C'est l'histoire de la plus humaine et de la plus tragique des erreurs. Infidèle à l'homme, par fidélité à lui-même, Caligula consent à mourir pour avoir compris qu'aucun être [...] ne peut être libre contre les autres hommes (Camus, 1962).

Caligula est maître de sa propre mort. En plus, il pousse les autres à la révolte, mais c'est une révolte qui se retourne contre lui donc qui le conduit à son propre suicide. C'est parce qu'il a compris le mécanisme fou qu'il a engendré qu'il consent à mourir. Il a compris " qu'on ne peut être libre contre les autres hommes ", dit Camus dans la même préface. Et si Caligula crie en mourant " Je suis encore vivant ", ce n'est pas par refus de la mort, mais parce qu'il veut incarner, pour la postérité, la révolte de l'Homme contre l'absurde. C'est un cri de défi: la survie qu'il prédit est aussi celle de la soif d'absolu qui le poussait et que tout homme peut retrouver en soi. Toujours dans sa révolte face à l'absurde, Caligula va se révolter contre le destin en provoquant et en laissant se produire une mort qu'il a lui-même choisie. II ne réagit pas quand les patriciens le préviennent d'un complot. Mourir est sa vraie volonté. Mais avant de mourir, il veut provoquer le chaos et dénoncer l'absurde. Une question inopinée peut en fait se poser: n'y aurait-il pas un sentiment altruiste sous-jacent à toute cette tyrannie? Caligula est-il vraiment un meurtrier?

Caligula comprend finalement qu'il n'a pas choisi la bonne voie et que sa "liberté n'est pas bonne ", sa mort doit donc être plutôt vue comme un sacrifice. C'est le meurtre de Caesonia qui marque le sommet de sa logique insensée et qui le ranime vers sa philanthropie perdue: l'empereur a compris son échec. Désormais, il lui apparaît que sa vie doit être immolée, c'est même une nécessité s'il veut que l'exemple de son entreprise marque les esprits. Le miroir dans lequel il se regarde lui fait se confronter à sa nouvelle personnalité pour laquelle il est " plein de haine ". Il ne se supporte plus alors, et brise l'accessoire. Il prouve ainsi le refus de ce qu'il est devenu, et témoigne sa volonté de participer à sa mort, de changer son assassinat en mort sacrificielle. C'est en quelque sorte sa dernière chance de rester la figure emblématique d'un ${ }^{2}$ homme qui aimait son prochain et qui s'était remis en question suite à ses fautes. Ainsi, il pourra rentrer dans l'histoire emblématique d'un homme qui aimait son prochain et qui s'était remis en question suite à ses fautes. Ainsi, il pourra rentrer dans l’histoire.

Lui qui a vécu déchiré par son impossible amour pour les hommes, amour perverti par la démesure et la logique et si semblable à la haine, voici qu'il meurt réconcilié. Ce destin fait de lui une figure dionysiaque et un héros tragique selon la définition qu'en donnait Nietzsche dans La Naissance de la tragédie (Crochet, 1973, p. 153).

Caligula a fait l'expérience de l'absurde qui lui a permis de sortir de l'ignorance; il veut maintenant instruire les hommes; il refuse de les laisser dans leur ignorance parce qu'il aimerait leur éviter la souffrance qu'il a lui-même ressentie à la mort de Drusilla. Mais pour cela, il ne faut pas que la vie humaine ait de l'importance, il ne faut pas que les hommes s'attachent à la vie et au monde. C'est ce que signifient ces paroles qu'il adresse à Cherea : " Le mensonge n'est jamais innocent. Et le vôtre donne de l'importance aux êtres et aux choses. [...] Ce monde est sans importance et qui le reconnaît 
Şengül KOCAMAN - Çukurova University Faculty of Education Journal, 43(1), 2014, 99-110

conquiert sa liberté » (Camus, 1958, p.37-38). C'est bien d'amour qu'il est question ici; I'amour qui va pourtant se manifester sous la forme d'une folie meurtrière à laquelle personne n'échappera.

C'est donc bien une noble préoccupation qui le motive: l'amour des hommes (Levi-Valensi, 2002, $p$. 78).

\section{Conclusion}

Caligula est une pièce qui met en scène un roi confronté à l'imminence de la mort. Devant une telle vérité, son pouvoir est aussi impuissant que celui du commun des mortels. Camus dit lui-même que son personnage « récuse l'amitié et l'amour, la simple solidarité humaine, le bien et le mal. Il prend au mot ceux qui l'entourent, il les force à la logique, il nivelle tout autour de lui par la force de son refus et par la rage de destruction où l'entraîne sa passion de vivre "(Camus, 1962). Dans le but de se révolter contre le destin, il recherche l'impossible, renverse toutes les valeurs, s'octroie la liberté extrême. En bref, Caligula nous présente un roi qui se moque de tout ce qui donne un sens à la vie comme l'amour, l'art, la religion, et qui ensuite se laisse assassiner en montrant que la mort est de toute façon absurde et que tout le monde est d'avance condamné. Peut-être que le mensonge et le cynisme sont nécessaires à notre survie. Caligula défend ainsi l'impossibilité de vivre avec la dure réalité: comment vivre dans un monde où tout n'est que mensonge?

\section{Bibliographie}

Bastien, S. (2006). Caligula, le texte. Caligula et Camus Interférences transhistoriques. New-York: Édition Rodopi.

Bartfeld, F. (1988). L'effet tragique; essai sur le tragique dans l'œuvre de Camus. Paris-Genève: Champion-Slatkine.

Camus, A. (1958). Caligula. Paris: Gallimard.

Camus, A. (1962). Préface à l'édition Américaine. Théâtre, récits, nouvelles. Paris: Gallimard.

Camus, A. (1985). Le Mythe de Sisyphe. Paris: Gallimard.

Chapoutot, J. (2006). Le jeune homme et la mort: nihilisme, logique de l'absurde et sens de l'amour dans le Caligula d'Albert Camus. Retrieved March 22, 2011, from http://www.sens-public.org/

Crochet, M. (1973). Les Mythes dans I'œuvre de Camus. Paris: Editions Universitaires.

Durand, A. Caligula; drame ne quatre actes d'Albert Camus. Retrieved May, 16, 2011, from http://www.comptoirlitteraire.com/317-camus-caligula-.html

Guerry, A. (2009). Note sur Camus et Suétone: la source ancienne de deux passages des carnets. Révolte contre le destin dans Caligula d'Albert Camus. Retrieved August, 22, 2011, from http://guerry.org/alain/textes/Seminaires\%202004-2011/2009-Th\%e9\%e2treR\%e9volte\%20contre\%20le\%20destin\%20dans\%20Caligula\%20d'Albert\%20Camus-Grand.pdf

Hobby, F. (1998). La Symbolique d'euphémisation dans I'univers fictif d'Albert Camus. New-York: Peter Lang Publishing.

Houel, F. (2006). Etudes sur Caligula. Paris: Ellipses.

Gay- Crosier, R. (1992). Caligula ou le paradoxe du comédien absurde. Albert Camus et le théâtre: actes du colloque tenu à Amiens en 1988. J. Lévi-Valensi (Ed.) Paris: Imec.

Levi-Valensi, J. (2002). Albert Camus et le mensonge: actes du colloque organisé par la Bibliothèque publique d'informations-Centre Pompidou, les 29 et 30 décembre, Paris. 
Şengül KOCAMAN - Çukurova University Faculty of Education Journal, 43(1), 2014, 99-110

Nguyen-Van-Huy, P. (1962). La métaphysique du bonheur chez Albert Camus. Neuchâtel, Suisse: Baconnière.

Onimus, J. (1965). Camus face au mystère. Paris: Editions Desclée De Brower.

Toura, H. (1992). Caligula devant le sacré: identification aux dieux-mères. Albert Camus et le théâtre: Actes du colloque tenu à Amiens en 1988. J. Lévi-Valensi (Ed.). Paris: Imec. 